\title{
BIENESTAR PSICOLÓGICO EN ESTUDIANTES DE LA UNIVERSIDAD NACIONAL DE MAR DEL PLATA, ARGENTINA, SEGÚN LA EDAD Y EL GÉNERO
}

\begin{tabular}{l} 
PSYCHOLOGICAL WELL-BEING OF STUDENTS (IN RELATION TO SEX AND GENDER) \\
FROM UNIVERSIDAD NACIONAL DE MAR DEL PLATA, ARGENTINA \\
\hline
\end{tabular}

Recibido: 08 de Septiembre del 2014 | Aceptado: 28 de Noviembre del 2014

ALICIA MASSONE ${ }_{1}$; SEBASTIÁN URQUIJO 2
(UNIVERSIDAD NACIONAL DEL MAR DEL PLATA, Mar del Plata, Argentina)

\begin{abstract}
RESUMEN
Objetivo: comparar el bienestar psicológico de los estudiantes de la Universidad Nacional de Mar del Plata, Argentina, en función de la edad y el género. Método: Se administró la Escala de Bienestar Psicológico de Ryff, (1989) adaptada al español por Van Dierendonck (2006) a una muestra no probabilística de 376 estudiantes regulares de diferentes carreras de la Universidad Nacional de Mar del Plata, $72 \%$ mujeres y $28 \%$ varones con una media de 24,8 años ( $D E=6,05$ años). Resultados: Las mujeres expresaron mayor autopercepción de Crecimiento Personal que los hombres $(U=11800, p=.01)$ y los adultos más que los jóvenes $(U=3942.00, p=.04)$. Conclusión: resultados podrían ser usados como insumos para el diseño de intervenciones tendientes a fortalecer las dimensiones del bienestar psicológico más deficitarias, para optimizar la disponibilidad de recursos personales y mejorar la trayectoria académica de los estudiantes.
\end{abstract}

PALABRAS CLAVE: Bienestar psicológico, edad, estudiantes universitarios, género.

\section{ABSTRACT}

Objective: To compare (considering sex and gender) the psychological well-being of students from Universidad Nacional de Mar del Plata, Argentina. Method: van Dierendonck's (2006) adaptation of Ryff"s (1989) Psychological Well-Being Scales (PWB) was applied to a non-probabilistic sample of 376 regular students enrolled in various undergraduate programs from Universidad Nacional de Mar del Plata (72\% women, $28 \%$ men), with a mean of 24,8 of age ( $D E=6,05$ years old). Results: Women expressed a higher self-perception of Personal Growth than men $(U=11800, p=.01)$, and adults than youngsters $(U=$ $3942.00, p=.04)$. Conclusion: These results can be considered relevant enough to design intervention programs oriented to consolidate the dimensions of psychological well-being with more deficits, to optimize the availability of personal resources and improve students' academic career.

KEY WORDS: Psychological well-being, age, college students, gender.

\footnotetext{
1. Afiliado a la Facultad de Psicología. CIMEPB. Universidad Nacional de Mar del Plata, Argentina. E-mail: amassone@mdp.edu.ar
}

2. Afiliado a la Facultad de Psicología. CIMEPB. Universidad Nacional de Mar del Plata, Argentina. E-mail: sebasurquijo@mdp.edu.ar 


\section{INTRODUCCIÓN}

Desde un enfoque salugénico, Westerhof y Keyes (2010) consideran el bienestar psicológico como criterio diagnóstico de salud mental, entienden la salud como la capacidad de cada persona de hacer 0 convertirse en lo que quiere ser y al bienestar como el resultado de la valoración global de elementos de naturaleza afectiva y cognitiva.

Desde este marco se considera importante la evaluación del bienestar psicológico (en adelante BP) en el ámbito universitario porque durante la trayectoria académica el estudiante vivencia numerosas situaciones de cambio en relación a sí mismo y al entorno, que inciden sobre su disponibilidad de recursos para enfrentar las circunstancias de la vida universitaria con éxito. Los estudios sobre BP evalúan el grado en que los estudiantes sienten satisfechos sus valores vitales y no tanto la frecuencia de actividades placenteras (Meléndez Moral, Tomas Miguel \& Navarro Pardo, 2008).

El BP es un fenómeno multidimensional mucho más amplio que la suma de emociones positivas a lo largo del tiempo que incluye aspectos sociales y del entorno, Ryff (1989) critica la idea de bienestar como ausencia de malestar 0 de emocionalidad negativa y propone un modelo multidimensional del BP, compuesto por seis dimensiones: autoconcepto, relaciones positivas, autonomía, dominio del entorno, satisfacción vital o propósito en la vida y crecimiento personal, que se evalúan de manera independiente (Keyes, 2002; Ryff et. al. 1989), a saber:

- Autoconcepto, refiere a tener actitudes positivas hacia uno mismo, es una característica fundamental del funcionamiento psicológico positivo (Keyes et. al. 2002).
- Relaciones positivas, es la capacidad de tener relaciones sociales estables y amigos íntimos (Ryff \& Singer, 1998).

- Autonomía, se asocia a la resistencia de la presión social y a la autorregulación del propio comportamiento, estar seguro de las propias convicciones y mantener independencia y autoridad personal (Ryff \& Singer, 2002).

- Dominio del entorno, es la habilidad personal para elegir y crear entornos favorables, para satisfacer los deseos y necesidades propios; las personas con buen dominio del entorno poseen mayor sensación de control y ejercen influencia sobre el contexto que los rodea.

- Crecimiento personal, alude a desarrollar al máximo las propias potencialidades y crecer como persona.

- Satisfacción vital o Propósito en la vida, es marcarse metas o planear objetivos que brindan sentido a la propia vida, se trata del componente indispensable del bienestar.

En relación al estudio de estas dimensiones del BP y su relación con el género, Ryff, Lee y Na (1993) afirman que las mujeres independientemente de su edad obtienen puntajes más altos que los hombres en Relaciones Positivas y en Crecimiento Personal, mientras que en Autoconcepto, Autonomía, Satisfacción vital y Dominio del entorno no expresan diferencias significativas. Algunos estudios contradicen estos resultados, Delfino y Zubieta (2011) hallaron en una muestra de estudiantes de la Universidad de Buenos Aires (Argentina), que las mujeres obtenían medias significativamente más altas que los hombres en las dimensiones Dominio del 
Entorno y Satisfacción vital. Barra (2010) encontró en universitarios chilenos diferencias significativas en Satisfaccion vital, las mujeres mostraron mayor puntaje que los hombres, este resultado concuerda con el de Vleioras y Bosma (2005) en universitarios griegos. Por el contrario, Roothman, Kirsten y Wissing, (2003) no encontraron diferencias significativas de género en ninguna de las dimensiones del BP en estudiantes universitarios de sudafricanos.

Según Garcia-Andalete (2013) los resultados de los estudios sobre BP son divergentes, no hay resultados concluyentes acerca de su vinculación con el género y la edad en estudiantes universitarios, por esto se plantea la necesidad de sumar estudios que aporten evidencias empíricas a las ya existentes. En función de los resultados controvertidos y contradictorios reportados en la bibliografía (Garcia-Andalete et. al. 2013; Roothman et. al. 2003; Zubieta, Muratori \& Fernández, 2012) este estudio se propone comparar cada una de las dimensiones del bienestar psicológico según las variables edad y género mediante un diseño descriptivo comparativo. Se considera que los resultados significan un aporte para el diseño de intervenciones tendientes a monitorear aquellas variables que inciden sobre el éxito en la trayectoria académica de los estudiantes universitarios.

\section{MÉTODO}

\section{Participantes}

Se conformó una muestra incidental no aleatoria de 376 estudiantes de la Universidad Nacional de Mar del Plata, Argentina, representativos de las diversas unidades académicas de la UNMdP, los porcentajes de estudiantes por facultad son: Arquitectura 9,3\%; Ciencias Económicas 9,3\%; Ciencias de la Salud 9\%; Derecho 7,2\%; Ciencias Exactas 5\%; Humanidades 9,6\%; Ingeniería $5 \%$ y Psicología $17 \%$ de la muestra total. Un $72 \%$ mujeres y $28 \%$ varones entre 18 y 62 años, con una edad promedio de 24,8 años y DS. $=6,05$; la distribución de género es proporcional a la distribución que se da en la Universidad.

\section{Procedimiento}

Se consideró como criterio de inclusión que los estudiantes tengan más de $50 \%$ de las asignaturas de la carrera aprobadas, voluntad de participar en el estudio y firmar un consentimiento informado. La administración de la escala se realizó en forma individual y autoadministrada, en un tiempo promedio de 30 minutos y en las aulas donde habitualmente desarrollan la actividad académica. Estuvo a cargo estudiantes avanzados de la carrera Licenciatura en Psicología, bajo supervisión de docentes investigadores.

\section{Instrumentos}

Para evaluar Bienestar Psicológico se administró la Escala de Bienestar Psicológico de Ryff (1989) cuenta con un total de seis sub-escalas y 29 ítems a los que se responde con un formato de respuesta Likert con puntuaciones comprendidas entre 1 (totalmente en desacuerdo) y 6 (totalmente de acuerdo).

La adaptación española de Van Dierendonck et. al., (2006), mantiene e incluso mejora los niveles de consistencia obtenidos por la escala original, con valores Alpha de Cronbach de 0,84 a 0,70. En la muestra bajo estudio el estadístico Alpha de Cronbach's para cada una de las subescalas fue: Autoconcepto (alpha .72), Relaciones Positivas (alpha .63), Autonomía (alpha .58), Crecimiento Personal (alpha .68), Satisfacción Vital (alpha .70) y Dominio del Entorno (alpha .67).

\section{RESULTADOS}

Para el análisis estadístico se usó el programa SPSS 17.0 para Windows, se analizó la normalidad de los datos mediante Kolmogorov-Smirnov, arrojó valores inferiores a 0.05 en todas las variables. Se 
calcularon los estadísticos descriptivos y para comparar cada una de las dimensiones del BP en función de edad y género, se aplicó la prueba $U$ de Mann-Whitney. Se codificaron los participantes según la edad en Jóvenes: entre 19 y 29 años y Adultos: 30 a 45 años.

En la Tabla 1 se exponen puntuaciones medias, máximas, mínimas y desvío standard, de la muestra total.

TABLA 1.

Estadísticos descriptivos de la escala para la muestra total.

\begin{tabular}{lcccc}
\hline & Media & DS & Mínimo & Máximo \\
& $n=376$ & & 8 & 24 \\
Autoconcepto & 18.43 & 3.16 & 10 & 24 \\
Relaciones Positivas & 16.22 & 2.34 & 15 & 37 \\
Autonomía & 25.46 & 3.67 & 8 & 21 \\
Dominio Entorno & 15.18 & 2.34 & 9 & 24 \\
Crecimiento Personal & 17.45 & 2.34 & 10 & 30 \\
Satisfacción Vital & 24.20 & 3.55 & & \\
\hline
\end{tabular}

En la Tabla 2 se exponen los promedios y el estadístico de contraste $U$ Mann-
Whitney según los grupos de edad, para cada dimensión del BP.

TABLA 2.

Promedios, desvío standard y comparación de las dimensiones del BP para los grupos de 19 a 29 y de 30 a 45 años de edad.

\begin{tabular}{|c|c|c|c|c|c|c|c|}
\hline & $\begin{array}{c}\text { Edad en } \\
\text { años }\end{array}$ & Media & D.S. & Std. Error & $\begin{array}{c}\text { Mann- } \\
\text { Whitney U }\end{array}$ & Z & $\begin{array}{c}\text { Asymp. Sig } \\
\text { (2-tailed) }\end{array}$ \\
\hline \multirow{2}{*}{ Autoconcepto } & 19 a 29 & 18.38 & 3.18 & .17 & & & \\
\hline & 30 a 45 & 18.93 & 2.821 & .51 & 4566.00 & -.85 & .39 \\
\hline Relaciones & 19 a 29 & 16.27 & 2.39 & .13 & \multirow[b]{2}{*}{4522.50} & & \\
\hline Positivas & 30 a 45 & 15.83 & 1.80 & .32 & & -.91 & .35 \\
\hline \multirow{2}{*}{ Autonomía } & 19 a 29 & 25.38 & 3.62 & .19 & & & \\
\hline & 30 a 45 & 26.06 & 3.34 & .61 & 4490.00 & -.99 & .32 \\
\hline Dominio del & 19 a 29 & 15.17 & 2.32 & .12 & & & \\
\hline Entorno & 30 a 45 & 15.33 & 2.64 &, 48 & 4880.50 & -.22 & .82 \\
\hline Crecimiento & 19 a 29 & 17.36 & 2.34 & .12 & & & \\
\hline Personal & 30 a 45 & 18.30 & 2.40 &, 43 & 3942.00 & -2.00 & $.04^{*}$ \\
\hline Satisfacción & 19 a 29 & 24.16 & 3.56 & ,19 & & & \\
\hline Vital & 30 a 45 & 24.73 & 3.39 & .61 & 4617.00 & -.76 & .44 \\
\hline
\end{tabular}

Existen diferencias significativas entre los jóvenes y los adultos en la dimensión Crecimiento personal $(U=3942.00, p=.04)$, los participantes de 30 a 45 años mostraron mayor percepción de crecimiento personal que los participantes de 19 a 29 años. No se detectaron diferencias significativas en
Autoconcepto, $(U=4566.00, p=.39)$, Relaciones Positivas, $(U=4522.50, p=$ .35); Autonomía, $(U=4490.0, p=.32)$, Dominio del Entorno, $(U=4880.5, p=.82)$, y Satisfacción Vital, $(U=4617.0, p=.44)$. 
En la Tabla 3 se presenta la comparación según sexo para cada dimensión del bienestar.

TABLA 3.

Comparación de las dimensiones del BP entre mujeres y hombres.

\begin{tabular}{lccccc}
\hline & \multicolumn{2}{c}{ Media (D.S.) } & Mann-Whitney U & Z & $\begin{array}{c}\text { Asymp. Sig. } \\
\text { (2-tailed) }\end{array}$ \\
\hline Autoconcepto & $18.43(3.06)$ & $18.48(3.41)$ & 13708.00 & -.26 & .78 \\
\hline Relaciones Positivas & $16.15(2.25)$ & $16.41(2.56)$ & 13260.50 & -.61 & .54 \\
\hline Autonomía & $25.56(3.62)$ & $25.20(3.80)$ & 13297.50 & -.70 & .47 \\
\hline Dominio del Entorno & $15.17(2.39)$ & $15.20(2.21)$ & 13545.50 & -.33 & .73 \\
\hline $\begin{array}{l}\text { Crecimiento } \\
\text { Personal }\end{array}$ & $17.67(2.26)$ & $16.88(2.48)$ & 11800.00 & -2.33 & $.01^{*}$ \\
\hline Satisfacción Vital & $24.38(3.35)$ & $23.74(4.03)$ & 13055.00 & -.96 & .33 \\
\hline Nota:* $\mathrm{p}<0.05$ & & & & \\
\hline
\end{tabular}

Se observó diferencias significativas entre hombres y mujeres en la dimensión Crecimiento Personal $(U=11800, p=.01)$, las mujeres mostraron un rango mayor en crecimiento personal que los hombres y no se expresaron diferencias significativas en las otras dimensiones del BP: Autoconcepto, $(U=13708.00, p=.78)$, Relaciones Positivas, $(U=13260.50, p=$ .54); Autonomía, $(U=13297.50, p=.47)$, Dominio del Entorno, $(U=13545.50, p=$ .73), y Satisfacción Vital, $(U=13055.00, p=$ .33).

\section{DISCUSIÓN}

En función de los resultados la dimensión del BP más sensible a la edad y al género es el Crecimiento Personal.

\section{Género y Bienestar Psicológico}

En la muestra bajo estudio, las mujeres revelaron mayor percepción de Crecimiento Personal, podría atribuirse a que las mujeres experimentan mayor preocupación por desarrollar al máximo sus potencialidades y mejorar como persona, asimismo las mujeres tienden a desarrollar actividades congruentes con sus valores y se comprometen más con sus sentimientos que con el mandato social (Keyes et. al. 2002). Este resultado también fue reportado por Garcia-Andalete (2013); Ryff (1989); Ryff y Singer (2008); y Zubieta, Muratori y Fernández (2012).

\section{Edad y Bienestar Psicológico}

Los participantes adultos mostraron un Crecimiento Personal significativamente mayor que los jóvenes que se atribuye a que las personas de más edad establecen metas personales intrínsecas más que extrínsecas, es decir, directamente ligadas con sus sentimientos, intereses y deseos más profundos, coherentes con sus necesidades psicológicas básicas (Valverde, Torres, Rahona, \& Gómez; 2009). El estudio de Garcia-Andalete et. al., (2013) también reporta una media más alta de Crecimiento Personal en los participantes de 30-55 años que en los de 18-29 años. 
Los resultados mostraron que el género y la edad inciden sólo sobre la percepción subjetiva del crecimiento personal, en tanto, no se observaron diferencias en las dimensiones Autoconcepto, Relaciones Positivas, Autonomía, Dominio del Entorno, y Satisfacción Vital, podría atribuirse a que el bienestar psicológico como lo entiende Carol Ryff tiene un fuerte componente cognitivo, cuya principal característica es ser estable, mantenerse en el tiempo e impregnar la vida global de los sujetos; mientras que el componente afectivo es mucho más lábil, momentáneo y cambiante (Casullo \& Solano, 2000).

También puede atribuirse a que se trabajó sobre una muestra bastante homogénea porque todos los participantes fueron estudiantes de la misma universidad, por lo tanto pertenecen a un contexto socio/cultural similar.

En conclusión, este estudio hace una contribución interesante porque la medición y evaluación del Bienestar Psicológico aporta información sobre la satisfacción de las necesidades psicológicas básicas (autonomía, dominio del entorno y relaciones positivas) y además un sistema de metas congruente y coherente (satisfacción vital, crecimiento personal y autoconcepto) de nuestros estudiantes universitarios. También estos resultados podrían ser usados como insumos para el diseño de intervenciones tendientes a fortalecer las dimensiones del bienestar psicológico más deficitarias, para optimizar la disponibilidad de recursos personales y mejorar la trayectoria académica de los estudiantes.

Este trabajo podría mejorarse ajustando algunas cuestiones asociadas a las características limitadas de la muestra, su falta de representatividad y a la necesidad de validación de la escala de bienestar psicológico de Ryff en población Argentina.

\section{REFERENCIAS}

Barra, E. (2010). Bienestar psicológico y orientación de rol sexual en estudiantes universitarios. Terapia psicológica, 28(1), 119-125.

Casullo, M. M., \& Solano, A. C. (2000). Evaluación del bienestar psicológico en estudiantes adolescentes argentinos. Revista de Psicología, 18(1), 35-68.

Delfino, G. I., \& Zubieta, E. M. (2011). Valores y política: Análisis del perfil axiológico de los estudiantes universitarios de la ciudad de Buenos Aires (República Argentina). Interdisciplinaria, 28(1), 93114.

García-Andalete, J. (2013). Bienestar psicológico, edad y género en universitarios españoles//Psychological well-being, age, and, gender among spanish undergraduates. Salud \& Sociedad, 4(1), 48-58.

Keyes, C. L. (2002). The mental health continuum: From languishing to flourishing in life. Journal of health and social behavior, 43, 207-222.

Meléndez Moral, J. C., Tomás Miguel, J. M., \& Navarro Pardo, E. (2008). Análisis del bienestar en la vejez según la edad. Revista Española de Geriatría y Gerontología, 43(2), 90-95.

Ryff, C. (1989). Happiness is not everything or is it? Explorations on the meaning of psychological well-being. Journal of Personality and Social psychology, 57, 1069-1089.

Ryff, C. \& Keyes. L. (1995). The structure of psychological well-being revisited. Journal of Personality and Social Psychology, 69, 719-727.

Ryff, C. (1995). Psychological well-being in adult life. Current directions in psychological science, 4, 99-104.

Ryff, C. D., \& Singer, B. (1998). The contours of positive human health.Psychological inquiry, 9(1), 1-28.

Ryff, C., \& Singer, B. (2002). From social structure to biology. Handbook of positive psychology, 63-73. 
Ryff, C. D., \& Singer, B. H. (2008). Know thyself and become what you are: A eudaimonic approach to psychological well-being. Journal of Happiness Studies, 9(1), 13-39.

Ryff, C. D., Lee, Y. H., \& Na, K. C. (1993). Through the lens of culture: Psychological well-being at midlife. Comunicación presentada en el encuentro de la Gerontological Society of America, New Orleans, LA.

Roothman, B., Kirsten, D. K., \& Wissing, M. P. (2003). Gender differences in aspects of psychological wellbeing. South African Journal of Psychology, 33(4), 212-218.

Van Dierendonck, D., Abarca, A. B., Díaz, D., Jiménez, B. M., Gallardo, I., Valle, C., \& Carvajal, R. R. (2006). Adaptación española de las escalas de bienestar psicológico de Ryff. Psicothema, 18(3), 572-577.

Valverde, C. V., Torres, G. H., Rahona, J. J., \& Gómez, D. (2009). Bienestar psicológico y salud: aportaciones desde la psicología positiva. Anuario de psicología clínica y de la salud= Annuary of Clinical and Health Psychology, 5, 15-28.

Vleioras, G., \& Bosma, H. A. (2005). Are identity styles important for psychological well-being?. Journal of adolescence, 28(3), 397-409.

Westerhof, G. J., \& Keyes, C. L. (2010). Mental illness and mental health: The two continua model across the lifespan. Journal of Adult Development, 17(2), 110-119.

Zubieta, E., Fernández, O., \& Sosa, F. (2012). Bienestar, valores y variables asociadas. Boletín de Psicología, 106, 7-27.

Zubieta, E., Muratori, M., \& Fernandez, 0. (2012). Bienestar subjetivo y psicosocial: explorando diferencias de género//Subjective and psychosocial well-being: exploring gender differences. Salud \& Sociedad, 3(1), 66-76. 